\title{
Efecto de la incorporación de Zeolita natural y Mucuna pruriens en el cultivo de arroz (Oryza sativa L.) bajo condiciones controladas en Upala, Alajuela, Costa Rica
}

\author{
${ }^{1}$ Liseth Sandoval Fernández, ${ }^{2}$ Andrés Zúñiga Orozco. ${ }^{3}$ Alexander Mendoza Luna, \\ ${ }^{2}$ Karla Montero Jara, ${ }^{2}$ Wagner Peña Cordero
}

1. Ingeniería Agronómica, Universidad Estatal Distancia, Sabanilla, Costa Rica: 1sandovalf@uned.ac.cr

2. Cátedra de gestión sostenible del suelo Escuela de Ciencias Exactas y Naturales. Universidad Estatal a

Distancia. Apartado Postal 474-2050. San Pedro, San José. Costa Rica. azunigao@uned.ac.cr ,

kmonteroj@uned.ac.cr, wpena@uned.ac.cr.

3. CONARROZ.. San Pedro, San José, Costa Rica. amendoza@ conarroz.com

Recibido: 18 de Mayo de 2020

Aceptado: 30 de Junio de 2020

\section{RESUMEN}

Se utilizó una fuente de abono verde, Mucuna pruriens a una dosis de $120 \mathrm{~g} / \mathrm{m}^{2}$ y una fuente de Zeolita mineral con dosis de $152 \mathrm{~g} / \mathrm{m}^{2}$, en combinación con fertilizante sintético para determinar la mejor combinación en cuanto a rendimiento de arroz obtenido o si aplicando como fuente única, podía lograr un incremento significativo en el rendimiento. Se logró determinar que el tratamiento con Mucuna pruriens+ fertilización convencional ( $<<0.1)$ fue el que mejor obtuvo rendimiento, además con una calidad de grano aceptable, también debido al uso de la técnica con el isótopo $15 \mathrm{~N}$ se determinó que contribuyó en una utilización eficiente del nitrógeno en la planta y un aporte significativo de materia orgánica al suelo. Otros efectos y factores involucrados bajo las condiciones de este ensayo son discutidos.

Palabras claves: Abonos verdes, Mucuna, Zeolita, Arroz, Nitrógeno.

\section{ABSTRACT}

Effect of the incorporation of natural Zeolite and Mucuna pruriens in the cultivation of rice (Oryza sativa L.) under controlled conditions in Upala, Alajuela, Costa Rica

A source of green manure, Mucuna pruriens at a dose of $120 \mathrm{~g} / \mathrm{m}^{2}$ and a source of Zeolite mineral at a dose of 152 $\mathrm{g} / \mathrm{m}^{2}$, in combination with synthetic fertilizer, were used to determine the best combination in terms of rice yield obtained or if applying as a single source, could achieve a significant increase in yield. It was possible to determine that the treatment with Mucuna pruriens + conventional fertilization $(\mathrm{p}<0.1)$ was the one that best obtained yield, in addition to an acceptable grain quality, it also, due to the use of the technique with $15 \mathrm{~N}$ isotope it was determined that it contributed to an efficient use of nitrogen in the plant and a significant contribution of organic matter to the soil. Other effects and factors involved under the conditions of this trial are discussed.

Key words: Green manure, Mucuna, Zeolite, Rice, Nitrogen. 


\section{Introducción}

El cultivo de arroz (Oryza sativa L.) es una fuente importante de alimento para una gran parte de la población mundial. Es vital para las mayores poblaciones del mundo y está profundamente relacionado con el patrimonio cultural de numerosas sociedades, es un rubro básico en la alimentación y mitigación de la pobreza en muchos países en vías de desarrollo (Romero 2015). Los países de mayor producción están en el continente Asiático: China, India, Indonesia, Bangladesh, Vietnam, Tailandia, Birmania y Japón, siendo los principales productores, en Europa: Italia, España, Rusia, Grecia y Portugal, en América: Estados Unidos, Brasil, Colombia, Perú y Argentina y África: Egipto, Nigeria, Madagascar y Costa de Marfil, el éxito como primera planta alimenticia de Asia se debe al hecho que el arroz es una especie mucho más productiva que otros cereales por una parte permite realizar varias cosechas cada temporada, por otra parte la productividad por hectárea cultivada es superior (CEI-RD 2011).

Para Costa Rica el arroz es parte de la canasta básica de alimentos, es la principal base de la nutrición en muchos hogares, siendo de gran importancia para el sector económico, productores arroceros y seguridad alimentaria, por lo cual es uno de los cultivos donde tiene que ser producido con un alto rendimiento por hectárea. Para el cultivo de arroz a nivel nacional se identificaron 58.539,7 ha sembradas durante el año agrícola, siendo este el cultivo anual con más extensión; los cantones que registraron mayor área sembrada fueron Liberia con 9.208,3 ha, Upala con 8.019,3 ha y Bagaces con 5.347,8 ha; la extensión sembrada de arroz en hectáreas por tipo de fertilizante: químico: 51.324,1, orgánico 68,5, utilización de ningún tipo de fertilizante 909,3 y combinación de orgánico con químico 6.238,0 (INEC 2015), con lo anterior se denota el gran área que se aplica en fertilización y por consiguiente las grandes cantidades que se ocupan para obtener un alto rendimiento en producción.

Para la producción del cultivo se toma en cuenta algunos factores como suelo, clima, paquete tecnológico o insumos, entre otros factores que van de la mano, dentro del uso de insumos en el cultivo del arroz, la fertilización compone un factor importante en la obtención de altos rendimientos, constituyendo un factor primordial en la producción nacional, la fertilización del cultivo normalmente se maneja con la aplicación de Nitrógeno (N), Fosforo (P), Potasio (K), Azufre (S) y Zinc (Zn) (Molina y Rodríguez 2012). Pero la eficiencia de estos fertilizantes va a depender en su mayoría del tipo de suelo y su manejo, existiendo pérdidas debido a la eficiencia típica de cada sitio, por lo anterior, los rendimientos potenciales se ven afectados. Uno de los motivos más recurrentes y de mayor peso es la afectación en la eficiencia del uso del Nitrógeno (N) (Mora et al, 2014), por lo cual se plantea el uso de coadyuvantes a nivel de suelo que puedan ayudar a retener los nutrimentos en el cultivo de arroz.

Debido a lo anterior, se analiza la utilización de Zeolita natural y Mucuna sp. como coadyudantes de suelo, en el caso de la Zeolita natural la estructura está integrada por una red tridimensional surcada por una trama interna de poros y cavidades, y por dos unidades: la primaria y la secundaria es la más simple y consiste de un tetraedro de cuatro iones de oxígeno que rodean un ion central de sílice ( $\mathrm{Si}$ ) o aluminio (Al), en la agricultura trae beneficios de mejorar la eficiencia de aprovechamiento de los fertilizantes al evitar las pérdidas por volatilización y lixiviación, la aplicación de Zeolita como aditivo a los fertilizantes reduce de $20-40 \%$ la cantidad necesaria de estos para el adecuado desarrollo de los cultivos, por lo que disminuye significativamente el costo de la fertilización (Paredes et al, 2013).

En el caso de la Mucuna sp. se vincularía directamente como un coadyuvante de suelo natural, sin embargo, tiene la ventaja de que aporta nutrientes, especialmente nitrógeno. Especies de abonos verdes de sistemas radiculares profundos ejercen un papel importante en el reciclaje de otros nutrientes desde las capas profundas del suelo hacia las capas más superficiales (Fonseca 2014). Experimentar con Zeolita y Mucuna sp. como alternativas disponibles para el mejoramiento de la disponibilidad de los nutrientes en cultivo de arroz es relevante para los productores a nivel nacional; que logren integrarlo a las prácticas de manejo como posible alternativa de fertilización sostenible.

En fertilización de suelos la eficiencia se refiere a que solo una parte del nutrimento aplicado al suelo es aprovechada por la planta (Kass 2007), en pocas palabras una parte de la fertilización se pierde y queda en disposición para contaminar potencialmente fuentes o cuerpos de agua y ecosistemas naturales.

A raíz de esto la importancia de realizar pruebas no solo reside en el mejoramiento de la eficiencia, sino también en brindar una reducción de la contaminación por fertilizantes y ayudar al suelo en la disponibilidad de nutrientes, además de tratar de reducir los costos de operación. Los nutrimentos que liberan los fertilizantes aplicados al suelo se pierden por lixiviación o porque reaccionan con otros materiales presentes en el suelo y precipitan, lo que los hace no disponibles para las plantas (Kass 2007). Con la investigación se pretende evaluar la absorción de nutrientes al incorporar Zeolita natural y Mucuna pruriens en el cultivo de arroz, durante etapas tempranas de crecimiento, como opción para mejorar el rendimiento del cultivo y de esta forma beneficiar a los productores de arroz, al ambiente y consumidores, esto porque se vería reflejado una disminución en los costos de producción y por ende una reducción en los precios de ventas. 


\section{Metodología}

La investigación se llevó a cabo en la provincia de Alajuela, cantón de Upala, distrito de Delicias, propiamente en el lugar llamado Santa Clara, ubicada en las coordenadas geográficas latitud 10.932291 , longitud $-85^{\circ} 082350$, sobre $49 \mathrm{msnm}$, con una temperatura máxima promedio anual de $32,2^{\circ} \mathrm{C}$, la mínima promedio anual es $19,5{ }^{\circ} \mathrm{C}$, la humedad relativa promedio mensual durante los meses de marzo y abril, alcanza su valor promedio más bajo de 78,0 y 76,2\% respectivamente, y sube a $88,8 \%$, la precipitación promedio anual $2395,1 \mathrm{~mm}$.

Se caracterizaron parcelas (21 parcelas), de 2x2 m (1x1 m parcela efectiva), dedicadas a la producción de arroz en secano durante la época lluviosa con el objetivo de evaluar la absorción de nutrientes al incorporar Zeolita natural y Mucuna pruriens en el cultivo de arroz, durante etapas tempranas de crecimiento, como opción para mejorar el rendimiento del cultivo. Para esta investigación se utilizó un diseño experimental de bloques completos al azar. La preparación del terreno fue realizada de acuerdo con las necesidades y requerimientos del cultivo de arroz en secano de acuerdo a las prácticas agronómicas implementadas por CONARROZ, antes de la siembra fue tomada una muestra unificada de las 21 parcelas para un análisis de suelo del tipo químico completo, materia orgánica y $\mathrm{N}$ total en una muestra compuesta. La forma de obtenerlo fue, tomando 10 submuestras de cada parcela (repetición) y se unificó, al final del experimento se tomó nuevamente una muestra, pero individualmente para los 7 tratamientos.

Con respecto al manejo agronómico del cultivo implementado fue según lo indicado por los investigadores de CONARROZ (Corporación Nacional del Arroz). La siembra fue según protocolos y procedimientos establecidos por CONARROZ. La variedad escogida es: PALMAR-18 a una densidad de 2 qq semilla/ha. Para una correcta y adecuada realización de la investigación se supervisó la aplicación de fertilizantes, específicamente en los tratamientos 2, 4, 5 y 7 debido a que eran los tratamientos en los que se tenían que incorporar el manejo agronómico usual en cuanto a fertilización de la zona seleccionada. La cantidad de fertilizante que fue aplicado fue equivalente al área de cada parcela $\left(4 \mathrm{~m}^{2}\right.$ por parcela de $2 \mathrm{x} 2$ m). Durante la investigación se desarrollaron 7 tipos de tratamientos: T1: Zeolita; T2: Zeolita + fertilización utilizada por CONARROZ; T3: Mucuna pruriens; T4: Mucuna pruriens + fertilización utilizada por CONARROZ; T5: Zeolita + Mucuna pruriens + fertilización utilizada por CONARROZ; T6: Zeolita + Mucuna pruriens; T7: Testigo comercial (fertilización utilizada por CONARROZ). Para cada tratamiento se usaron de 3 repeticiones, quedando 21 parcelas de $4 \mathrm{~m}^{2}$ para un total de $84 \mathrm{~m}^{2}$ de parcela experimental efectiva $\left(194 \mathrm{~m}^{2}\right.$ en total) con un borde de $1.5 \mathrm{~m}$.

En las parcelas de $4 \mathrm{~m}^{2}$, se marcó un área central de $1 \mathrm{x} 1 \mathrm{~m}$, la cual fue delimitada para la aplicación de ${ }^{15} \mathrm{~N}$ y fue el área efectiva para evaluar las variables dependientes. Para la determinación del contenido de nitrógeno se utilizó isotopos estables en el caso ${ }^{15} \mathrm{~N}$, donde la aplicación se efectúo al momento de siembra en una dosis de $1 \mathrm{~g} / \mathrm{m}^{2}$ en cada parcela (en el área de 1x1m), la aplicación del ${ }^{15} \mathrm{~N}$ fue, determinar el contenido de nitrógeno. En los tratamientos 3, 4, 5 y 6 la incorporación de la $M$. pruriens fue a una dosis de: $300 \mathrm{~kg} / \mathrm{ha}$ en peso seco lo que equivale a $30 \mathrm{~g} / \mathrm{m}^{2}$ para cada repetición. Antes de la incorporación fue limpiada el área de arvenses, se pesó la cantidad por aplicar y se aplicó cuando el suelo estuvo a Capacidad de Campo, esto por cuanto debe favorecerse el proceso de mineralización con la humedad. En el caso de los tratamientos 2 , 4, 5 y 6 en los que fue aplicada la Zeolita natural: esta aplicación se realizó una sola vez en la primera fertilización comercial a una dosis de: $152 \mathrm{~kg} /$ ha lo que equivale a $152 \mathrm{~g} / \mathrm{m}^{2}$ para cada repetición.

En la etapa fenológica R4, se tomó análisis foliar individual por cada tratamiento y repetición. En la misma etapa fenológica se tomaron muestras para la determinación de los niveles de nitrógeno con ${ }^{15} \mathrm{~N}$. Para calcular el \% de $\mathrm{N}$ en la planta derivada del fertilizante aplicado (\%Npdf) se utilizó la siguiente ecuación:

$(\% \mathrm{Npdf}=\% \mathrm{~N}$ en la planta derivado del fertilizante $)$

(1) $\% \mathrm{Npdf}=\frac{\%{ }^{15} \mathrm{~N} \text { en exceso de la muestra }}{\%{ }^{15} \mathrm{~N} \text { en exceso del fertilizante }} \times 100$

$\%{ }^{15} \mathrm{~N}$ en exceso de la muestra $=\%{ }^{15} \mathrm{~N}$ muestra $-0,366$

$\%{ }^{15} \mathrm{~N}$ en exceso del fertilizante $=\%{ }^{15} \mathrm{~N}$ del fertilizante $-0,366$

Después, se procedió a calcular el $\mathrm{N}$ en la planta derivado del suelo ( $\% \mathrm{Npds}=\% \mathrm{~N}$ en la planta derivado del suelo $)$, que es el $\mathrm{N}$ total de la planta $(100 \%)$ deduciendo el $\mathrm{N}$ en la planta derivado del fertilizante:

$\% \mathrm{Npds}=100-\% \mathrm{Npdf}$

QNpdf = cantidad de $\mathrm{N}$ en la planta derivado del fertilizante

$\mathrm{QNpdf}=\frac{\% \mathrm{Npdf} \times \mathrm{N}_{\mathrm{T}}}{100}$

La cosecha de la granza en la parcela o área de evaluación se realizó de forma manual, con el fin de obtener material para evaluar de la calidad molinera y rendimiento en toneladas por hectárea. Las variables dependientes medidas fueron las 
siguientes: Rendimiento, Calidad de Grano, Contenido de elementos en el suelo, Absorción de nutrientes, ${ }^{15} \mathrm{~N}$, las variables independientes fueron, M. pruriens y Zeolita.

Finalmente, a los resultados se le realizó un ANDEVA y dado que se compararon categorías y no dosis variables, se aplicó la prueba de Duncan con un error experimental de: $10 \%$ (90\% de confiabilidad) lo anterior por ser una prueba pequeña y de corte preliminar. En el caso de las variables dependientes se realizó comparaciones descriptivas con gráficos y tablas mediante hoja de cálculo en Excel®.

\section{Resultados}

$\Rightarrow \quad$ Determinar los cambios en $\mathrm{pH}$, acidez, CICE, \%SA, nutrimentos, relación $\mathrm{C} / \mathrm{N}$, materia orgánica y nitrógeno total en el suelo cultivado con arroz al ser enmendado con $M$. pruriens y Zeolita natural, mediante análisis químico de suelo.

$\Rightarrow$

En el cuadro 1 se observa una leve disminución generalizada en pH, excepto en el testigo. En acidez hay incrementos leves, excepto en el T3 y T7. En CICE hay leves variaciones entre los tratamientos. En \%SA solo se observa un leve incremento en el T3, lo que coincide con los niveles de acidez encontrados al final del experimento en el T3 (M. pruriens), lo cual indica que el uso de $M$. pruriens aplicada sola tiende a acidificar el suelo levemente. La relación $\mathrm{C} / \mathrm{N}$ se incrementa en todos los tratamientos, especialmente los T3, T4 y T5, en los cuales el denominador común fue usar M. pruriens. Finalmente, para el valor de materia orgánica fue muy evidente que todos los tratamientos incrementaron sus contenidos excepto el testigo y los incrementos con menor magnitud se obtuvieron donde solo se aplicó zeolita (T1) o zeolita con fertilización (T2).

Cuadro 1. Comparativo en valores iniciales y finales en las variables $\mathrm{pH}$, acidez, CICE, \%SA, C/N y \% MO en el suelo de los diferentes tratamientos. Alajuela, Costa Rica. 2017

\begin{tabular}{|c|c|c|c|c|c|c|c|c|c|c|c|c|}
\hline \multirow{3}{*}{ Tratamientos } & \multirow{2}{*}{\multicolumn{2}{|c|}{$\begin{array}{c}\text { pH } \\
6.5-7.5\end{array}$}} & \multirow{2}{*}{\multicolumn{2}{|c|}{$\begin{array}{c}\text { Acidez } \\
\text { 0.3-1 }\end{array}$}} & \multirow{2}{*}{\multicolumn{2}{|c|}{$\begin{array}{c}\text { CICE } \\
\begin{array}{c}5-25 \\
\mathrm{cmol}(+) / \mathrm{L}\end{array}\end{array}$}} & \multirow{2}{*}{\multicolumn{2}{|c|}{$\begin{array}{c}\% \mathrm{SA} \\
10-30 \%\end{array}$}} & \multirow{2}{*}{$\begin{array}{l}\mathrm{C} / \mathrm{N} \\
8-20\end{array}$} & \multirow{2}{*}{\multicolumn{3}{|c|}{$\begin{array}{l}\% \mathrm{MO} \\
2-5 \%\end{array}$}} \\
\hline & & & & & & & & & & & & \\
\hline & Inicial & Final & Inicial & Final & Inicial & Final & Inicial & Final & Inicial & Final & Inicial & Final \\
\hline T1 & & & & & & 23,97 & & 0,5 & & 8,5 & & 4,0 \\
\hline & & 5,5 & & 0,13 & & & & & & & & \\
\hline $\mathbf{T} 2$ & & 5,7 & & 0,14 & & 25,84 & & 0,5 & & 8,9 & & 3,9 \\
\hline $\mathbf{T 3}$ & & 5,5 & & 0,17 & & 23,33 & & 0,7 & & 8,8 & & 4,2 \\
\hline T4 & 5,8 & 5,6 & 0,13 & 0,13 & 23,95 & 22,88 & 0,5 & 0,6 & 8,4 & 9,1 & 3,6 & 4,0 \\
\hline T5 & & 5,6 & & 0,12 & & 23,36 & & 0,5 & & 9,2 & & 4,5 \\
\hline T6 & & 5,6 & & 0,13 & & 24,26 & & 0,5 & & 9,4 & & 4,2 \\
\hline T7 & & 5,9 & & 0,12 & & 23,18 & & 0,5 & & 8,5 & & 2,9 \\
\hline
\end{tabular}

$\Rightarrow \quad$ Determinar el contenido de nutrimentos presentes en el cultivo de arroz de los distintos tratamientos en la etapa fenológica de la fase R4 en Santa Clara de Upala mediante un análisis foliar, para observar la cantidad de nutrimentos absorbidos por la planta y comparar los contenidos totales entre tratamientos. 
Como se puede observar en el cuadro 2, el N, Zn y Mn no hubo diferencias significativas en contenidos totales acumulados en el suelo al final del experimento. Para $\mathrm{Ca}, \mathrm{Mg}, \mathrm{K}, \mathrm{P}, \mathrm{Cu}$ y $\mathrm{Fe}$ si hubo diferencias significativas. En cuanto a los contenidos inicial versus final por cada nutrimento, podemos decir que no se observaron cambios significativos en los contenidos en $\mathrm{N}$ y $\mathrm{Cu}$, en el resto de los nutrimentos si se observaron cambios significativos. Para Ca, los contenidos disminuyeron en todos los tratamientos excepto en T1 y T2, siendo el T2 (zeolita + fertilización) sobresaliente en aumento. Para Mg, hubo un aumento en todos los tratamientos excepto en 4 y 7, siendo este último el testigo, en el cual era esperable esa disminución. Para K, el comportamiento es variable, mientras el T1, T4 y T5 bajaron, los T2, T3, T6 y T7 subieron, estos últimos por el denominador común que fue la presencia de zeolita. Para $\mathrm{P}$ y $\mathrm{Zn}$, todos los tratamientos incrementaron los niveles, excepto el testigo. Para $\mathrm{Mn}$ y Fe, todos los tratamientos disminuyeron los niveles. Finalmente, para $\mathrm{Cu}$, todos los tratamientos aumentaron levemente o se mantuvieron igual, excepto en el testigo. Podemos concluir que en la mayoría de los casos los nutrimentos en el tratamiento testigo mantuvieron los niveles más bajos al final del experimento, tal es el caso de $\mathrm{N}, \mathrm{P}, \mathrm{Zn}, \mathrm{Mn}, \mathrm{Cu}$ y Fe, además en otros nutrimentos como Ca y Mg fueron también dentro de la categoría más baja, solamente en el elemento K hubo un incremento muy marcado, posiblemente debido a la aplicación del fertilizante no aprovechada.

Cuadro 2. Comparativo de nutrimentos en el suelo, inicio versus final del experimento. Alajuela, Costa Rica. 2017

\begin{tabular}{|c|c|c|c|c|c|c|c|c|c|c|}
\hline \multirow[b]{2}{*}{ Nutrimento } & \multirow[b]{2}{*}{ Unidad } & \multirow[b]{2}{*}{$\begin{array}{c}\text { Rangos } \\
\text { óptimos* }\end{array}$} & Tratamientos & 1 & 2 & 3 & 4 & 5 & 6 & 7 \\
\hline & & & Inicial & \multicolumn{6}{|c|}{ Final } & \\
\hline $\mathbf{N}$ & $\%$ & $0.5-0.2$ & 0,30 & 0,33 & 0,31 & 0,33 & 0,31 & 0,34 & 0,31 & 0,24 \\
\hline Ca & & $4-15$ & 18,45 & 18,47 & 19,86 & 17,53 & 17,59 & 17,76 & 18,38 & 17,57 \\
\hline Mg & $\operatorname{cmol}(+) / \mathrm{L}$ & $1-6$ & 5,02 & 5,11 & 5,46 & 5,20 & 4,85 & 5,15 & 5,36 & 4,91 \\
\hline $\mathbf{K}$ & & $0.2-0.8$ & 0,35 & 0,26 & 0,38 & 0,43 & 0,31 & 0,33 & 0,39 & 0,58 \\
\hline & & $10-50$ & 14 & 19 & 19 & 24 & 19 & 15 & 21 & 12 \\
\hline $\mathbf{P}$ & & & & & & & & & & \\
\hline Zn & $\mathrm{mg} / \mathrm{L}$ & $2-10$ & 5,4 & 6,6 & 5,8 & 6,4 & 6,5 & 5,8 & 5,7 & 3,6 \\
\hline Mn & & $5-50$ & 121 & 73 & 77 & 70 & 77 & 79 & 72 & 45 \\
\hline $\mathrm{Cu}$ & & $1-20$ & 12 & 13 & 13 & 13 & 12 & 13 & 12 & 9 \\
\hline $\mathrm{Fe}$ & & $10-50$ & 241 & 198 & 179 & 240 & 215 & 184 & 212 & 114 \\
\hline
\end{tabular}

Los contenidos de $\mathrm{N}$ están por debajo de lo recomendado (Cuadro 3), los tratamientos que se presentan con mejores contenidos son T2, T4 y T7, Para P, los contenidos son adecuados, siendo el T1, T3 y T6 los de mejor contenido, Para K, los contenidos son adecuados, siendo el T1 el de menor contenido, Para Ca y Mg los contenidos son muy similares y son adecuados, Para S, los contenidos están llegando al límite inferior, excepto el T1, T3 y T6, En el caso del Zn, se presentaron contenidos similares llegando al límite superior, Para $\mathrm{Cu}$, se presentaron contenidos similares llegando al límite inferior, Para el caso de Mn, los valores van desde 273 a 406 ppm, siendo el T2 el de mayor contenido y el T6 el de menor, Para Fe, los contenidos van desde 161,7 a 309,7 siendo el T1 el de mayor contenido y el T6 el de menor, Finalmente, para el caso del $\mathrm{B}$, los contenidos fueron levemente superiores a lo recomendado, el T2 fue el más alto.

Cuadro 3. Contenidos de nutrimentos foliares en la etapa R4 de crecimiento. Alajuela, Costa Rica. 2017

\begin{tabular}{|c|c|c|c|c|c|c|c|c|c|}
\hline \multirow[t]{2}{*}{ Nutrimento } & \multirow[t]{2}{*}{ Unidad } & \multirow{2}{*}{$\begin{array}{c}\text { Rangos } \\
\text { optimos } * * * *\end{array}$} & \multicolumn{7}{|c|}{ Tratamiento } \\
\hline & & & 1 & 2 & 3 & 4 & 5 & 6 & 7 \\
\hline \multirow[t]{2}{*}{$\mathbf{N * *}$} & $\%$ & $2,6-4,8$ & & & & & & & \\
\hline & $\%$ & & 1,2 & 1,7 & 1,1 & 1,7 & 1,6 & 1,2 & 1,7 \\
\hline $\mathbf{P} *$ & $\%$ & $0,1-0,4$ & & & & & & & \\
\hline \multirow[t]{2}{*}{$\mathbf{K}^{*}$} & $\%$ & $1-3,5$ & 0,4 & 0,2 & 0,4 & 0,3 & 0,2 & 0,4 & 0,2 \\
\hline & $\%$ & & 2,2 & 2,6 & 2,7 & 2,5 & 2,8 & 2,4 & 2,5 \\
\hline $\mathbf{C a} *$ & & $0,2-4,0$ & 0,25 & 0,30 & 0,19 & 0,28 & 0,26 & 0,21 & 0,26 \\
\hline $\mathbf{M g}^{*}$ & & $0,15-0,7$ & 0,15 & 0,17 & 0,14 & 0,18 & 0,16 & 0,15 & 0,17 \\
\hline $\mathbf{S}^{*}$ & & $0,15-0,30$ & 0,23 & 0,19 & 0,24 & 0,18 & 0,19 & 0,22 & 0,17 \\
\hline $\mathbf{Z n}^{* *}$ & ppm & $18-50$ & 49,0 & 48,3 & 51,3 & 42,0 & 49,7 & 46,0 & 44,3 \\
\hline $\mathbf{C u}^{*}$ & ppm & $7-20$ & 7,3 & 10,3 & 8,3 & 9,3 & 11,0 & 7,3 & 8,7 \\
\hline
\end{tabular}




\begin{tabular}{cccccccccc}
\hline $\mathbf{M n}^{* *}$ & $\mathrm{ppm}$ & $40-800$ & 286 & 406 & 336 & 343 & 366 & 273 & 308 \\
$\mathbf{F e}^{*}$ & $\mathrm{ppm}$ & $75-300$ & 309,7 & 218,3 & 226,0 & 196,0 & 191,7 & 161,7 & 195,7 \\
$\mathbf{B}^{*}$ & $\mathrm{ppm}$ & $<4$ & 4,7 & 5,3 & 4,0 & 4,7 & 4,3 & 4,0 & 4,7 \\
\hline
\end{tabular}

$* \mathrm{p}<0,1$ en contenidos finales

$* * \mathrm{p}>0,1$ en contenidos finales

$\Rightarrow \quad$ Estimar el contenido de nitrógeno acumulado en el cultivo de arroz a través de la técnica isotópica ${ }^{15} \mathrm{~N}$, en la etapa fenológica R4 de la planta, para observar la absorción de nitrógeno y comparar los contenidos totales entre tratamientos

En la prueba isotópica se determinó que el tratamiento que contenían una mayor cantidad de nitrógeno con diferencias significativas ( $p>0,10)$ fue el T1 (Zeolita) y el T4 (Mucuna sp, + fertilización) con menor presencia de nitrógeno. Por otro lado, en cuanto a $\mathrm{N}$ total, el T4 es quien presenta el mayor contenido de $\mathrm{N}$ total $(\mathrm{p}>0,10)$. En la figura 1 se muestran los contenidos de $\mathrm{N}$ total durante la fase R4 de crecimiento durante el experimento, acá se observa que uno de los mejores tratamientos en rendimiento, el T4, acumula mayor contenido, lo anterior indica que, las mayores acumulaciones de $\mathrm{N}$ foliar presentaron mejor rendimiento (figura 2).

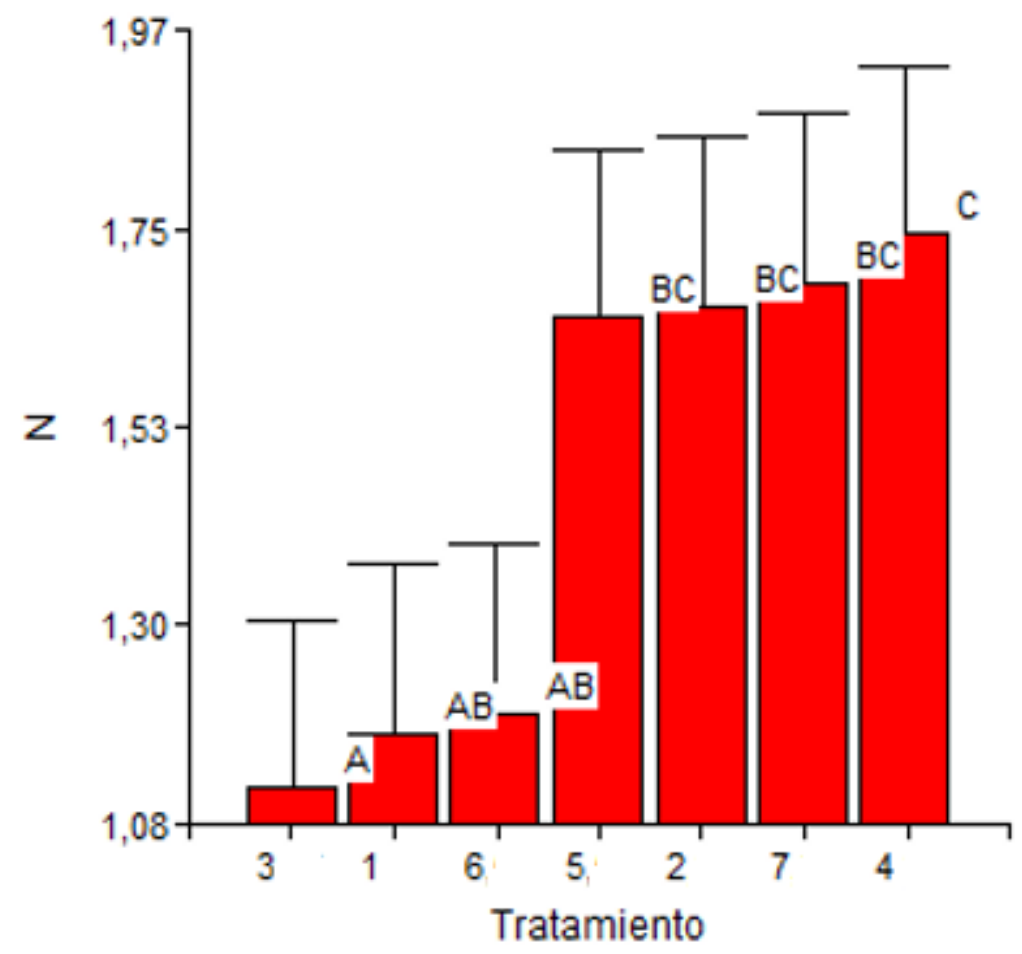

Figura 1. Absorción de nitrógeno total (\%) durante el desarrollo de la planta en la fase R4 del estado fenológico de la planta, Alajuela, Costa Rica, 2017.

Como se observa en el cuadro 4, hubo mejores resultados de la eficiencia de $\mathrm{N}$ en el T6 (Zeolita + M. pruriens) con un $\%$ de aprovechamiento de 23,2\%, a pesar de que el T4 (M. pruriens + Fertilización) presentó mayor cantidad de N total en las plantas, lo anterior hace suponer que, en el T4, se relacione más el contenido de N Total con el rendimiento ya que, fue el más productivo; sin embargo, no fue el más eficiente, esto hace suponer que la $M$. pruriens como abono verde requiere mejores condiciones para el intercambio iónico, tal como sucede en presencia de materiales como zeolita o fertilizantes. Por otro lado, es importante señalar que los tratamientos en los que se utilizó únicamente zeolita, acompañada con fertilización nitrogenada o con $M$. pruriens, mostraron un mayor aprovechamiento (cuadro 4) y una mayor recuperación de $\mathrm{N}$, en comparación con los demás tratamientos utilizados.

Cuadro 4, Aplicación de M. pruriens y zeolita, con o sin fertilización comercial en la utilización de nitrógeno marcado con 
${ }^{15} \mathrm{~N}$ y aplicado como urea, en el cultivo de arroz (Oryza sativa)

\begin{tabular}{|c|c|c|c|c|}
\hline Tratamientos & $\%$ Npdf & QNPdf (N/ha)* & Ap \% & $\mathrm{NT}^{*}$ \\
\hline T3: M.pruriens & 0,381 & $0,25 \mathrm{a}$ & 5,4 & $65,6^{\mathrm{a}}$ \\
\hline T4: M.pruriens+Fertilización & 0,265 & $0,26 \mathrm{a}$ & 5,8 & $104,3 b$ \\
\hline T7: Testigo (Fertilización) & 0,806 & $0,57 \mathrm{a}$ & 12,5 & $71,4^{\mathrm{a}}$ \\
\hline T6: Zeolita+M.pruriens & 1,652 & $0,53 \mathrm{a}$ & 23,2 & $68,3^{\mathrm{a}}$ \\
\hline T1: Zeolita & 0,862 & $0,53 \mathrm{a}$ & 11,5 & $63,1^{\mathrm{a}}$ \\
\hline T2: Zeolita+Fertilización & 0,527 & $0,50 \mathrm{a}$ & 10,9 & $97,7^{\mathrm{a}}$ \\
\hline T5: Zeolita+M.pruriens+Fertilización & 0,583 & $0,53 \mathrm{a}$ & 11,5 & $90,8^{\mathrm{a}}$ \\
\hline
\end{tabular}

*letra diferente indica diferencias significativas

$\Rightarrow \quad$ Determinar la productividad del cultivo del arroz a través del rendimiento y calidad molinera del grano con respecto a cada tratamiento realizado para comprobar la factibilidad de la utilización de Zeolita natural y Mucuna sp,

En esta variable se determinó que el tratamiento de mayor rendimiento fue el T4 (M.pruriens + fertilización) y con menor rendimiento el T7: testigo comercial $(\mathrm{p}<0,1)$

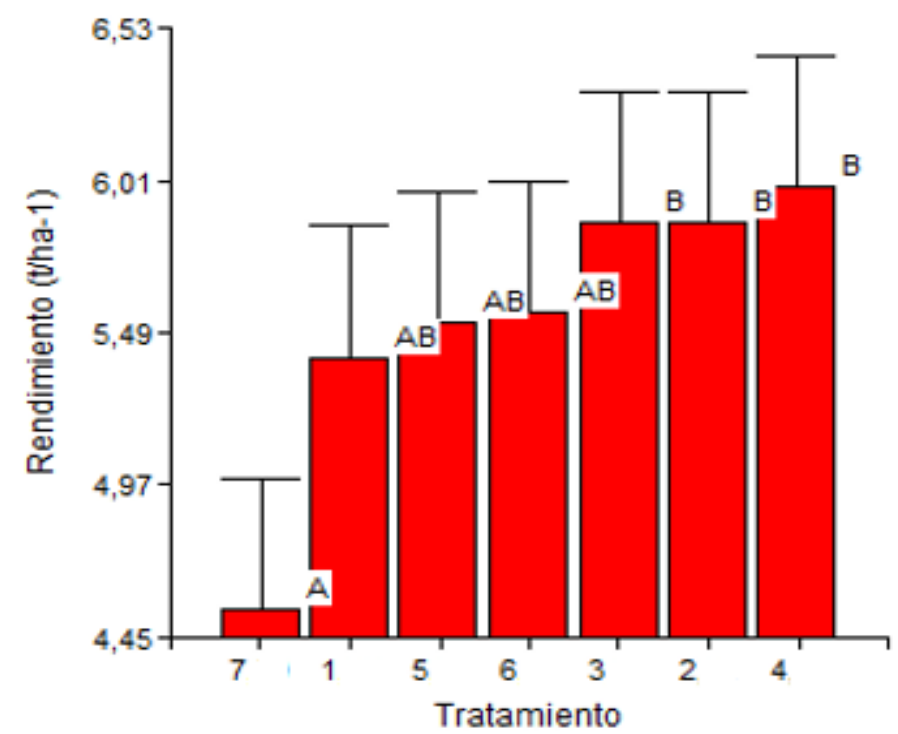

Figura 2. Rendimiento del cultivo de arroz, variedad PALMAR-18, en los diferentes tratamientos efectuados, Alajuela, Costa Rica, 2017.

$\Rightarrow \quad$ Evaluar el efecto de la utilización de Zeolita natural y M. pruriens en cuanto a rendimiento y calidad del cultivo de arroz,

En cuanto a rendimiento, se determinó la calidad molinera (calidad de grano), la mejor calidad encontrada fue el T3 ( $M$. pruriens) y con menor calidad el T7 (testigo comercial). 


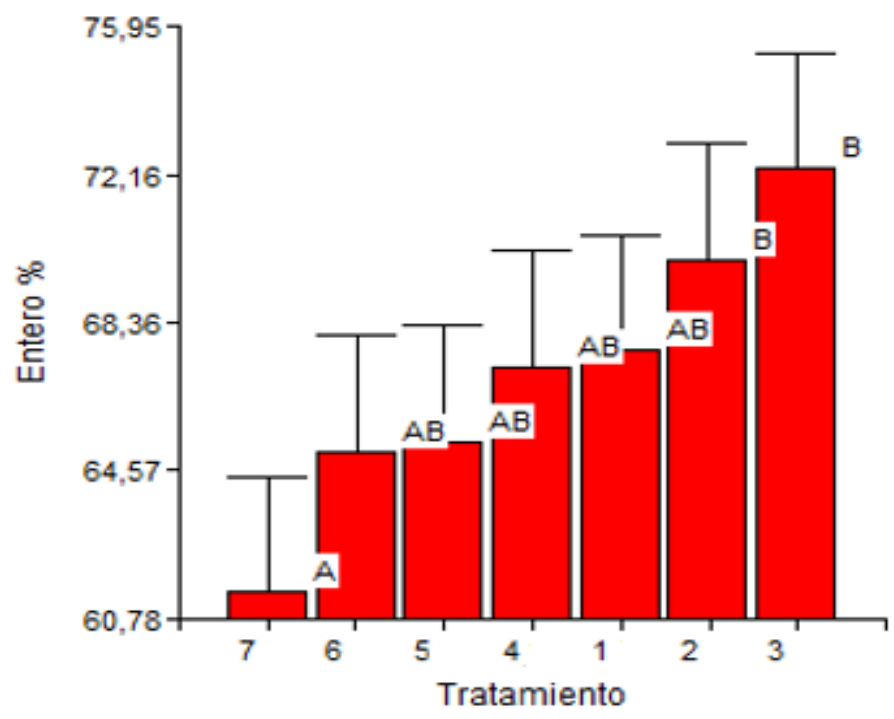

Figura 3. Calidad molinera del cultivo de arroz, variedad PALMAR-18, en los diferentes tratamientos efectuados, Alajuela, Costa Rica, 2017.

\section{Discusión}

Con relación a los resultados de este estudio, las condiciones del suelo al inicio del ensayo fueron moderadamente fértiles, sin embargo, con contenidos bajos de materia orgánica. En el cuadro 1 se muestra las variaciones de pH, acidez, CICE, C/N y \%MO, las cuales siguen siendo adecuadas para el desarrollo del cultivo al final del experimento, sin embargo, se incorporó materia orgánica en todos los tratamientos excepto en el testigo, tal como se observa en el cuadro 1, cabe resaltar que los tratamientos con $M$. pruriens, se vieron más favorecidos (T3, T4, T5, T6), para el caso del T3 y T4 coincide con ser los tratamientos más productivos (figura 2) y para el T3 coincide como uno de los de mejor calidad molinera, por lo tanto, se concluye que incorporar materia orgánica al suelo es uno de los factor beneficioso en cuanto al valor productivo y de calidad de grano. La utilización de $M$. pruriens, contribuye mejorando la fertilidad del suelo por dos vías: fijación de nitrógeno atmosférico en el suelo y por el aporte de material vegetativo, el cual se transforma en materia orgánica, mejorando la parte física, química y biológica del suelo (CENTA 2012).

Otro de los tratamientos más productivos fue el T2 (Zeolita+ fertilización), para este caso específico es posible que, la aplicación de la Zeolita al momento de la siembra colaboró en la conservación de las características químicas del suelo como se muestra en el cuadro 2 y posiblemente en la conservación de la humedad, dicha característica no fue medida en este experimento, pero la zeolita retiene muchas veces su peso molecular en contenido de agua. Además, los tratamientos con zeolita presentaron una mayor recuperación y aprovechamiento de $\mathrm{N}$, tal y como se describió en el cuadro 4, según Obregón et al, (2015), las zeolitas son una familia de minerales aluminosilicatos que por su alta capacidad de intercambio catiónico y afinidad por el $\mathrm{NH}^{4+}$ se ha utilizado con éxito en la reducción del tránsito de $\mathrm{NO}^{3-}$ hacia la zona saturada en suelos arenosos. El uso de estos minerales trae múltiples beneficios, entre ellos, aumentos entre el 5 y $44 \%$ en el rendimiento de diferentes cultivos por ejemplo avena, trigo, así como la reducción del contenido de $\mathrm{NO}^{3-}$ en el lixiviado en relación con el $\mathrm{NH}^{4+}$ del suelo, de esta forma aumentando la capacidad de retención de nutrientes y por ende su aprovechamiento. La zeolita al ser un mineral soluble atrapa los nutrimentos y los pone al alcance de la planta, el cual evita que, por efecto del sol, exceso de agua y características adversas del suelo la formulación de elementos suministrados se pierda en un buen porcentaje o se fije antes de ser asimilado por la raíz (Cárdenas y Touma, 2011), Para el caso del T4, siendo el más productivo, se comprueba que fue el que mayor cantidad de $\mathrm{N}$ total acumuló (figura 1), aunque no fue el más eficiente en términos de uso del nutrimento (cuadro 4), caso contrario con el T6 quien presentó mayor eficiencia, pero no fue el más productivo.

Para el caso del Nitrógeno no hubo diferencias significativas en el suelo al final del experimento (cuadro 2), sin embargo, si se observa una mayor acumulación a nivel foliar en los tratamientos T2, T4, T5 y T7 (cuadro 3), para el caso del T4, ya se mencionó que no fue el de mejor aprovechamiento, pero incidió en un buen balance para obtener el mejor rendimiento. Sumado al aporte de nitrógeno, se presenta en el cuadro 1 la relación C/N en el suelo, siendo los tratamientos 6, 5 y 4 los más altos, aunque comparado con los contenidos recomendados fueron bajos. En estos tratamientos el denominador común fue la presencia de $M$. pruriens; e interpretando los valores Flores (2010) y Sanclemente et al, (2013), señalan que, cuando 
la relación $\mathrm{C} / \mathrm{N}$ es baja significa que hay mucho nitrógeno y poca energía, una parte del $\mathrm{N}$ liberado es tomado por los microorganismos y el resto es incorporado al suelo y puede ser absorbido por las plantas, Para el caso del experimento podemos interpretar que, aunque los valores globales fueron bajos, hubo una liberación pronta de $\mathrm{N}$ y a mediano y largo plazo se realizó un efecto benéfico en contenidos de $\mathrm{C}$ y $\mathrm{N}$ para sostener la fertilidad del suelo. Lo anterior aplicaría para implementar en el caso de los suelos agrícolas, ya que muchas veces, son suelos degradados, los cuales pierden materia orgánica y carbono al mismo tiempo, dando como resultado erosión en la parte química y microbiológica del suelo, para este caso señala Castro (2016) que con la incorporación de abonos verdes el rendimientos de cultivos ser mayores, incluso en intervalos cortos de tiempo entre incorporación del abono y siembra del cultivo, sobre todo en condiciones tropicales de altas temperaturas y humedad, las cuales contribuyen con la descomposición de la materia orgánica (Castro, 2016).

Relacionando la relación $\mathrm{C} / \mathrm{N}$ con el rendimiento, se observa como uno de los tratamientos con una relación $\mathrm{C} / \mathrm{N}$ alta, produjo el mejor rendimiento en el T4 (Mucuna sp, + fertilización), lo anterior indica posiblemente que, aunque el C aportado brindó buena cantidad de energía, el $\mathrm{N}$ aportado fue suficiente para ser absorbido por los microorganismos y por las raíces de las plantas en una cantidad justa y balanceada, la cual permitió a las plantas ser más productivas.

El aporte de $\mathrm{N}$ al cultivo en el mejor tratamiento (T4) y en el testigo comercial (T7) fue de 60,7 kg/ha, lo cual es muy positivo, dado que, con el aporte de $M$. pruriens se igualó el aporte del fertilizante, en otros estudios se ha reportado hasta $150 \mathrm{~g} / \mathrm{ha}$ de N/año (PASF 2017) y de $156 \mathrm{~kg} / \mathrm{ha}$ (Céspedes 2017, sin publicar), lo que significa un aporte orgánico considerable y disponible tanto a corto plazo como a largo plazo por la acumulación de $\mathrm{C}$ y $\mathrm{N}$ disponible.En cuanto al papel del P absorbido a nivel foliar, muestra diferencias significativas ( $<<0,1)$, siendo T1, T3 y T6 los de mayor absorción, según Amador (2012), el 25\% del fósforo absorbido se queda retenido en la paja y el resto queda en el grano, sin embargo, no hay relación clara del nutrimento y el rendimiento observado.

En cuanto al contenido foliar de $\mathrm{K}$ en el cultivo de arroz, se obtuvo diferencias significativas ( $\mathrm{p}<0,1)$, siendo el T5 y T3 los de mayor absorción, así como el T1 (Zeolita) el de menor cantidad de elemento absorbido, el T3 coincide como el tratamiento de mejor calidad por lo que es posible establecer una relación con el contenido de K; hay estudios que mencionan que cerca del $19 \%$ del potasio se va en el grano y el $81 \%$ queda retenido en la paja, además se reporta como un elemento esencial para el llenado de grano y calidad del mismo (Amador, 2012). Para el elemento Ca el comportamiento fue distinto, el T2 (Zeolita+ Fertilización) fue el que presentó mayor absorción, demostrando que la zeolita en este estudio resultó positivo, esto por cuanto, las Zeolitas son acondicionadores de la fertilidad química de los suelos, no así de la fertilidad biológica, sin embargo, se debe tener presente que las Zeolitas tienen algún efecto en las propiedades físicas del suelo, ya que, el uso de este material mejora la fluidez del agua por la reducción de la densidad aparente (Pérez, 2014).

Para los elementos Mg y S, estos elementos poseen diferencias significativas $(\mathrm{p}<0,1)$ en los contenidos absorbidos, en el caso del Mg, el que obtuvo mayor absorción fue el T4, lo cual coincide con el mejor rendimiento. Para el S hubo diferencias significativas $(\mathrm{p}<0,1)$, el T3 fue el de mayor contenido absorbido y coincide con ser el de mejor calidad de grano por lo que es posible establecer relación entre el elemento y la calidad. La absorción de magnesio y azufre está involucrada en la producción de clorofila y por consiguiente es necesario para la síntesis de proteínas, incrementa la eficiencia en el uso del nitrógeno, y tiene efectos positivos sobre el rendimiento de las plantas (Kass 2007 y Chen 2017).Para los micronutrientes Zn y Mn, no hubo diferencias significativas, pero para el elemento B si las hubo ( $<<0,1)$ donde el T2 (Zeolita+ Fertilización) absorbió mayor cantidad, Estos elementos no dejan de ser importantes para la planta de arroz, según Kass (2007), las funciones e importancia que tienen en las plantas son: enzimáticas, en reacciones de reducción-oxidación, en síntesis, de clorofila, su participación en la fotosíntesis, en la influencia que tienen en el crecimiento de brotes nuevos y en la fijación de nitrógeno atmosférico. Finalmente, para el Hierro hubo las diferencias significativas ( $>0,1)$, siendo T1 el de mayor cantidad y 6 con menor cantidad.

En cuanto a la calidad molinera (grano entero), se obtuvo diferencias significativas ( $<<0,1)$, siendo el T3 (M. pruriens) el de mejor calidad y el T7 (Fertilización) el de menor calidad, con esto resalta el papel de la M. pruriens para favorecer la calidad molinera, esto por cuanto las prácticas de incorporación de abonos verdes y barbechos, mejoran las propiedades biológicas del suelo, es decir, incremento de materia orgánica y aumento de la diversidad de mesofauna en el suelo (Romo 2008).

\section{Conclusiones}

Cualquier tratamiento fue mejor al testigo (fertilización Comercial) en cuanto a rendimiento. Los mejores rendimientos se obtuvieron en los tratamientos 4 (M. pruriens + fertilización), 3 (M .pruriens) y 2 (Zeolita + fertilización), siendo el T4 el de mayor magnitud, incluso muy superior al rendimiento promedio de la zona $(3,5 \mathrm{ton} / \mathrm{ha})$.

En cuanto a calidad de grano, cualquier tratamiento fue mejor al testigo (fertilización comercial), Los mejores se obtuvieron en T3 (M. pruriens) y T2 (Zeolita + fertilización), éstos coinciden y se relacionan con lo obtenido en rendimiento, siendo los mejores junto al T4. 
A pesar que el aporte de $\mathrm{N}$ al cultivo en tratamiento (T4) y en el testigo comercial (T7) fue superior a los demás (60,7 kg/ha), es de suponer que el abono verde de $M$. pruriens se mantendrá a mediano o largo plazo, dado que, representa un factor importante para el mantenimiento del reservorio de la materia orgánica del suelo (Stevenson 1994).

De acuerdo con el uso de la técnica de medición isotópica con ${ }^{15} \mathrm{~N}$ se obtuvo que el T4, quien fue el tratamiento más productivo, acumuló mayor cantidad de $\mathrm{N}$ total, aunque no fue el más eficiente en términos de uso del nutrimento; caso contrario con el T6 (Zeolita+ M. pruriens) quien presentó mayor eficiencia, pero no se ubicó dentro de los tratamientos más productivos. Por lo tanto, la implementación de M. pruriens y Zeolita, en los planes de fertilización en el cultivo de arroz son muy necesarios para lograr una absorción balanceada de nutrientes en la planta y lograr mejores rendimientos, así como de calidad molinera.

Además de lo anterior el T4 no solo mejoró en cuanto a rendimiento, sino también en la capacidad de absorción de nutrimentos en la planta.

\section{Referencias}

Cárdenas D, Touma M .2011. Evaluación en el cultivo de arroz del efecto de las briquetas de urea con diferentes concentraciones de Zeolita en dos diferentes tipos de siembra: método al voleo y método de transplante en la zona Febres Cordero - Provincia de los Ríos (en línea). Ecuador. Consultado el 06 de octubre 2016. Disponible en: http://www,dspace,espol,edu,ec/handle/123456789/19161

Castro E. 2016. Utilización de leguminosas forrajeras como abonos verdes para la producción de cultivos forrajeros y leche en ganaderías doble propósito en el trópico seco (en línea). Colombia. Consultado el 22 de octubre 2017. Disponible en: http://www, bdigital,unal,edu,co/51526/1/79763436,2016,pdf

Centro de Exportación e Inversión de la República Dominicana, CEI-RD. 2011. Perfil Económico Arroz (en línea). República Dominicana. Consultado el 06 de octubre 2016. Disponible en: http://www,ceird,gov,do/estudios_economicos/estudios_productos/perfiles/arroz,pdf

Centro Nacional de Tecnología Agropecuaria y Forestal (CENTA). 2012. Abonos verdes (en línea). El Salvador, Consultado $\begin{array}{llllll}\text { el } & 09 & \text { de } & \text { octubre } & 2017 & \text { Disponible }\end{array}$ https://www,jica,go,jp/project/elsalvador/0603028/pdf/production/vegetable_11,pdf

Céspedes S; Zuñiga A; Mendoza A; Montero K; Peña W; Villalobos R. 2017. Evaluación de la incorporación de Mucuna pruriens y Crotalaria spectabilis, sobre el aporte y absorción de nutrientes en el cultivo de arroz (Oryza sativa), Datos sin publicar.

Chen, J. 2017. La función del magnesio en el cultivo de plantas (en línea). México. Consultado el 09 de setiembre 2017. Disponible en: http://www,pthorticulture,com/es/centro-de-formacion/la-funcion-del-magnesio-en-el-cultivo-deplantas/

Correndo, A; García, O. 2012. Archivo Agronómico \#14 (en línea). sI. Consultado el día: 6 de enero de 2018. Disponible en: http://www,ipni,net/publication/ialacs,nsf/0/2983F9B934DDE60D852579BA00747DF4/\$FILE/AA14,pdf

Fonseca, JA. 2014. Agricultura biológica. (en línea). Colombia. Consultado el 06 de octubre 2016, Disponible en: http://datateca,unad,edu,co/contenidos/301615/16,_Abonos_verdes,pdfInstituto

Flores, J. 2010. Agricultura Ecológica Manual y Guía Didáctica (en línea). España, Consultado el 12 de octubre 2017. Disponible en: http://www, fundesyram,info/biblioteca,php?id=3948

Instituto Nacional de Estadística y Censos, INEC. 2015. VI Censo Nacional Agropecuario Cultivos agrícolas, forestales y ornamentales (en línea). Costa Rica. Consultado el 06 de octubre 2016, Disponible en: http://www,inec,go,cr/system/files_force/documetos-bibliotecavirtual/01,_atlas_estadistico_agropecuario_2014,pdf

Kass, D. 2007. Fertilidad de suelos. San José, CR; EUNED.188 p,

Mora, S; Gavi, F; Tijerina, L; Pérez J; y Peña, JJ. 2014. Evaluación de la recuperación del nitrógeno y fósforo de diferentes fuentes de fertilizantes por el cultivo de trigo irrigado con aguas residuales y de pozo (en línea). México. Consultado el 06 de octubre 2016. Disponible en: http://www,scielo,org,co/pdf/acag/v63n1/v63n1a04,pdf

Molina, E. 2002. Análisis de suelo y su interpretación, Centro de Investigaciones Agronómicas, Costa Rica. Consultado el 25 de abril de 2018.2 Disponible en: http://www,infoagro,go,cr/Inforegiones/RegionCentralOriental/Documents/Suelos/SUELOSAMINOGROWanalisiseinterpretacion,pdf

Molina, E; Rodríguez, JH. 2012. Fertilización con N, P, K y S, y curvas de absorción de nutrientes en arroz var, CFX 18 En Guanacaste (en línea). Costa Rica. Consultado el 06 de octubre 2016, Disponible en: https://dialnet, unirioja,es/servlet/articulo?codigo=4056989

Obregón, N; Díaz, JE; Daza, MC; \& Aristizabal, HF. 2016. Effect of zeolite application on nitrogen recovery and maize yield, Acta Agronómica. 65(1): 24-30.

Paredes, R; Ramírez, MA, Osuna, E; Salvador, A; Gómez, P; Mandujano A. 2013. Zeolita natural: Alternativa ecológica y económica para la agricultura de temporal en México (en línea). México. Consultado el 06 de octubre 2016, 
Pérez, F. 2014. Evaluación de la Zeolita natural utilizada como una tecnología productiva y limpia, aplicada al pasto Maralfalfa (Pennisetum sp) como un complemento en el uso de los fertilizantes nitrogenados (en línea). Costa
Rica.
Consultado
el
06 de
octubre
2016.
Disponible
en: http://repositoriotec,tec,ac,cr/bitstream/handle/2238/3290/evaluacion_Zeolita_natural_utilizada_tecnologia_prod uctiva_limpia,pdf?sequence $=1$ \&isAllowed $=y$

Programa Amazonia Sin Fuego, (PASF). 2017. Cuadernillo de cultivo de Mucuna Negra, (en línea). Bolivia. Consultado el 06 de 0 octubre 2016, Disponible en: https://www,pasf,org,bo/docs/BUPNgLProuOOkNPbOPNixtZBWe6RIHg0,pdf

Romero, RL. 2015. Respuesta de diferentes poblaciones de Leptochloa spp a las aplicaciones de herbicidas de ACC asa utilizados en el cultivo de arroz (Oryza sativa L). en línea. Ecuador. Consultado el 06 de octubre 2016. Disponible en: http://repositorio,ug,edu,ec/bitstream/redug/7350/1/Tesis\%20malezas\%2028\%20Junio\%202015,pdf

Romo, DP. 2008. Efecto de cinco leguminosas como abonos verdes y cultivos de cobertura, para la producción de maíz duro (Zea mays L) en el sector de mascarilla-Carchi. en línea. Ecuador. Consultado el 10 de octubre 2017. Disponible en: dspace,pucesi,edu,ec/bitstream/11010/239/1/T72108,pdf

Sanclemente, R; Oscar Eduardo; Prager, M; Beltrán, LR. 2013. Aporte de Nitrógeno al suelo por Mucuna pruriens y su efecto sobre el rendimiento de maíz dulce (Zea mays L). en línea. Colombia. Consultado el 06 de octubre 2016. Disponible en: https://dialnet,unirioja,es/descarga/articulo/5344985,pdf 